CSF Leaks and Hearing Outcomes

\title{
Audiologic Improvement Following MCF Approach for Spontaneous Cerebrospinal Fluid Leaks
}

Mohamedkazim Alwani; Elhaam Bandali; Lauren Van Buren; Charles Yates; Rick Nelson

\begin{abstract}
Objective: To determine the audiologic improvement after middle cranial fossa (MCF) approach to repair spontaneous cerebrospinal fluid (SCSF) leaks

Study Design: Retrospective cohort study

Setting: Tertiary referral center

Patients: Twenty-four consecutive patients (27 ears) with temporal bone sCSF leak over a 4year period. Patient age, gender, ethnicity, body mass index (BMI), location of CSF leak, recurrence of CSF leak, and presence of encephalocele(s) were recorded.

Intervention: Audiometric testing in patients undergoing MCF repair of temporal bone sCSF leak.
\end{abstract}

Main Outcome Measures: Comparison of preoperative and postoperative pure tone average (PTA), air bone gap (ABG), and word recognition score (WRS) in the sCSF leak ear.

Results: Out of 27 ears, $55 \%$ had multiple tegmen defects and $82 \%$ had $\geq 1$ encephaloceles. There were no recurrent CSF leaks at a median follow up of 4 months. The mean (SD) preoperative PTA and ABG were 40.58 [15.67] dB and 16.44 [6.93] dB, respectively. There was significant improvement in mean PTA $(10.28$ [8.01] dB; $p<0.001$; Cohen $d=0.95)$ and ABG (9.31 [7.16] dB; P <0.001; Cohen $d=0.88$ ) after SCSF repair. Mean WRS improved (by 3.07 [6.11] \%; $p=0.024$; Cohen $d=0.46$ ) from a mean preoperative WRS of 93.16 [9.34] \% to a mean postoperative WRS of 96.26 [6.49] \%.

Conclusions: MCF approach for repair of sCSF leaks yields significant improvement in conductive hearing loss and is highly effective in management of the entire lateral skull base where multiple bony defects are often identified. 
CSF Leaks and Hearing Outcomes

\section{INTRODUCTION}

Spontaneous cerebrospinal fluid (SCSF) leaks of the temporal bone occur when there is both a bone defect around the pneumatized spaces of the mastoid or middle ear and a defect in the dura surrounding the brain. The resulting middle ear and mastoid cerebrospinal fluid along with coincidental encephalocele leads to conductive hearing loss in the SCSF leak ear. Thus, patients present with aural fullness, hearing loss and persistent middle ear effusion on exam. A high index of clinical suspicion is required for accurate diagnosis in addition to high resolution CT imaging to evaluate the tegmen of the skull base and clinical exam to rule out other causes. Ancillary diagnostic modalities to establish the diagnosis of a SCSF leak include myringotomy with fluid analysis for beta-2 transferrin, radionuclide studies, and magnetic resonance imaging (MRI).

Most patients with sSCF leaks are middle age (age 45-65 years), female, and obese(1). The incidence of sCSF leaks approximately doubled from 2002 to 2012, mirroring the rise in obesity that has occurred in the base 3-4 decades(2). Several mechanisms regarding the pathophysiology of SCSF leaks and tegmen dehiscence have been elucidated including isolated skull thinning(3), obstructive sleep apnea(4,5), and intracranial hypertension(3,6,7).

Surgical repair of the skull base (tegmen tympani and tegmen mastoidium) and the dura is the current standard treatment for SCSF leaks. The most common surgical approach is the middle cranial fossa (MCF) approach(1), while the transmastoid (TM) alone, and the combined TM/MCF approaches have also been employed(1). The advantages of the MCF approach include the ability to visualize the entire skull base floor without disruption of the ossicles and the low recurrence rate. Here we report the audiologic outcomes of patients who underwent MCF repair of sCSF leaks. All sCSF leak patients present with some degree of conductive hearing loss and thus, it is important to evaluate the improvement in conductive hearing loss after surgical repair. 
CSF Leaks and Hearing Outcomes

\section{METHODS}

\section{Study Approval}

After institutional review board approval (IRB \#1806954727), the Cerner database was searched for all patients who underwent MCF approach for repair of SCSF leaks by the senior authors (RFN and CWY) between January 2014 and December 2018 at our tertiary care center.

\section{Eligibility Criteria and Patient Selection}

The study inclusion criteria were as follows: age $\geq 18$ years, diagnosis of sCSF leak by senior author, those who underwent surgical repair and documented preoperative and postoperative audiometric assessments. At our institution, the MCF approach for surgical repair of SCSF leaks is exclusively used for repair of SCSF leaks. Diagnosis of sCSF leak was made with 1) clinical microscopic examination of the ear showing no evidence of cholesteatoma with clear middle ear fluid or clear pulsatile fluid coming from a pressure equalizing tube and 2) tegmen dehiscence on coronal views of high-resolution temporal bone CT imaging and the absence of ossicular or mastoid septa bone erosion. Beta 2 transferrin testing of the fluid and MRI imaging were only occasionally used for diagnosis. Specifically, high-resolution T2weighted MRI to check for a temporal encephalocele is only obtained at our institution when the diagnosis of SCSF leaks is uncertain. Determining the presences of an encephalocele on MRI preoperateively does not change our surgical management. Exclusion criteria included history of temporal bone trauma, presence of cholesteatoma, neoplasms, and prior cranial base surgery.

\section{Surgical and Reconstructive Technique}

The MCF approach to repair sCSF leaks has been described previously(8). Briefly, a pterional incision was performed with harvesting of a large temporalis fascia graft. The temporalis muscle was incised to leave a cuff of muscle and fascia attached to the calvarium and the muscle was reflected anteriorly. A 4.5 centimeter $(\mathrm{cm}) \times 4.5 \mathrm{~cm}$ craniotomy was performed over the middle ear and mastoid with the craniotomy centered over the root of the zygoma. Intraoperative mannitol $(0.5 \mathrm{~g} / \mathrm{kg})$ was administered and the patient was hyperventilated to end-tidal CO2 $<30$. 
The temporal lobe dura was elevated, and the dura and skull base defects were identified. The entire floor of the middle fossa was explored including the anterior petrous apex.

Encephaloceles were amputated and gently removed from the middle ear or mastoid without disruption of the ossicles or injury to the facial nerve. Reconstruction consisted of a multilayer closure with a fascia soft tissue seal on the skull base, split calvarial bone grafts over bone defects, and collagen matrix (DuraGen; Integra LifeSciences, Plainsboro, New Jersey and Durepair; Medtronic, Minneapolis, Minnesota) abutting the native dural defects. In more recent cases, tegmen bone defects were reconstructed with bone grafts and the entire floor was reconstructed with HydroSet calcium phosphate bone substitute (Stryker corp). Extradural temporalis fascia and collagen matrix were also included. Concurrent superior canal dehiscences were plugged with bone wax prior to skull base reconstruction with HydroSet bone cement. The craniotomy was replaced and the muscle and galeal layers were sutured in a water-tight fashion. Lumbar drains were not routinely used as this has not been shown to improve outcomes in temporal sCSF leaks(8).

\section{Clinical and Audiometric Data}

Demographic data collected included patient age, gender, and ethnicity. The body mass index (BMI) of patients at the time of surgery was also recorded. The authors tabulated the location of dehiscence, as well as the presence of concurrent meningoencephaloceles.

Audiometric data was collected preoperatively and postoperatively. Pure tone averages (PTA) were collected in accordance with the American Academy of Otolaryngology - Head and Neck Surgery minimal reporting guidelines(9), where by air conduction (AC) and bone conduction (BC) thresholds at $500 \mathrm{~Hz}, 1000 \mathrm{~Hz}, 2000 \mathrm{~Hz}$, and $3000 \mathrm{~Hz}$ were averaged. If the $3000 \mathrm{~Hz}$ frequency was not recorded, the average of the $2000 \mathrm{~Hz}$ and $4000 \mathrm{~Hz}$ was used. Airbone gaps (ABGs) were calculated by simple subtraction of average air conduction thresholds from average bone conduction thresholds. Word recognitions scores (percent correct) were recorded and were obtained at $40 \mathrm{~dB}$ above the speech reception threshold in most cases. 


\section{Statistical Analysis}

Data was aggregated using Microsoft Excel software, version 16.12 (Microsoft). All statistical analyses were performed using SPSS, version 24 (IBM). Demographic and select clinical data were summarized using summary statistics. Where indicated, data was tested for normality of distribution using the Shapiro-Wilk test. Comparison of continuous variables was achieved using 2-tailed paired sample t-test at $95 \%$ confidence. A p-value of $<0.05$ was considered statistically significant. Unit-free effect size was determined using Cohen $d$, whereby a $d$ value of 0.2 was considered to be a small effect, a $d$ value of 0.8 was considered to be a medium effect, and a $d$ value $\geq 0.8$ was considered to be a large effect. 
CSF Leaks and Hearing Outcomes

\section{RESULTS}

\section{Demographics}

A total of 34 records were identified on retrospective chart review. Only 27 records met inclusion criteria. Seven patients were excluded due to lack of postoperative audiograms. An overview of the demographic data is provided in Table 1. Three patients had bilateral procedures, resulting in 24 total patients. The mean (SD) age of the 24 patients in the study was 60.8 [9.2] years with male patients constituting $25 \%(n=6)$ and female patients constituting $75 \%(n=18)$. The mean (SD) BMl of the study population was $37.8(6.8) \mathrm{kg} / \mathrm{m}^{2}$. All patients underwent high resolution CT (HRCT) preoperatively and all patients had dehiscence of the tegmen (Figure 1). Of the 27 records of sCSF leaks included in the study, $55.55 \%(n=15)$ occurred on the right and $44.45 \%(n=12)$ occurred on the left. The cohort constituted of 20 (83.3\%) Caucasian patients and 4 (16.7\%) African American patients (Table 1). Only two patients in our cohort underwent beta 2 transferrin testing, with both tests yielding positive confirmatory results.

\section{Clinical and Surgical Findings}

Preoperatively 21 out of 27 patients $(77.78 \%)$ had documented complaints of hearing loss on the affected side. A total of 16 out of 27 cases $(59.26 \%)$ had both clear otorrhea and documented complaints of hearing loss on the affected side simultaneously. All cases reported either clear otorrhea or hearing loss on the affected side (Table 1). Physical exam revealed 15 ears with intact tympanic membranes (TM), 8 ears with pressure equalization tubes (PETs), and 4 ears with TM perforations without PETs. None of the patients with TM perforations or PETs received PET removals and/or tympanoplasty simultaneously during MCF approach or prior to the reported postoperative audiograms.

Table 1 also provides an overview of the surgical findings from our cohort. All sCSF leaks were repaired using a multilayer reconstruction of the tegmen via the MCF approach (Figure 1). In 5 ears (18.52\%), the dehiscent area was purely over the tegmen tympani, while 9 
ears (33.33\%) exhibited defects purely over the tegmen mastoideum. Concurrent defects of the tegmen tympani and tegmen mastoideum were noted in 13 ears (48.15\%). Single dehiscent areas were seen in 12 ears (44.44\%), while multiple areas of dehiscence were noted in 15 ears (55.56\%). Encephaloceles were seen to be concomitantly present in 22 ears $(81.48 \%)$. Surgical repair of SCSF leaks was successful (defined as absence of CSF otorrhea at 4-week postoperative visit) in all patients in the cohort.

\section{Audiometric Testing}

The median postoperative audiometric follow-up was 4 months with a range of 1 to 37 months (Table 1). An overview of all audiometric outcomes data is provided in Table 2. The mean (SD) preoperative PTA and mean (SD) preoperative ABG was 40.58 [15.67] and 16.44 [6.93], respectively. The mean (SD) postoperative PTA and mean (SD) postoperative ABG was 30.30 [15.62] and 7.13 [5.68], respectively. The mean (SD) PTA showed a significantly improvement after surgery of $10.28[8.01] \mathrm{dB}(\mathrm{p}<0.001$; Cohen $d=0.95)$.

ABG closure was noted in 26 out of 27 cases (96.30\%) with mean (SD) postoperative ABG improvement of 9.31 [7.16] $(p<0.001$; Cohen $d=0.88)$. Of the 26 cases that demonstrated ABG closure, 23 cases (88.46\%) exhibited ABG closure to $\leq 15 \mathrm{~dB}$. Additionally, the WRS showed a statistically significant improvement from a mean (SD) preoperative WRS of 93.16 [9.34] \% correct to a mean postoperative WRS of 96.26 [6.49] \% correct, yielding a mean improvement of 3.07 [6.11] \% correct $(p=0.024$; Cohen $d=0.46)$ (Table 1). Preoperative to postoperative comparisons of audiometric outcomes are summarized in Figures 2 and 3. One case demonstrated no change in ABG. This patient experienced persistent pneumocephalus without middle ear fluid after skull base repair at another institution. Preoperatively at our institution, the patient had a minimal $A B G$ and audiogram remained unchanged postoperatively (preoperative and postoperative ABG of $2.5 \mathrm{~dB}$ ). One patient has a retracted tympanic membrane and yellow serous otitis media at last follow-up. A PET was placed for eustachian 
tube dysfunction and two months later the patient had an open PET with a dry ear and a slightly improved ABG from preoperatively. 
CSF Leaks and Hearing Outcomes

\section{DISCUSSION}

The MCF approach is advantageous for the repair of temporal sCSF leaks for several reasons. Temporal bone SCSF leaks occur almost exclusively through the tegmen over the middle ear or mastoid or both. Posterior fossa sCSF leaks are extremely rare(10). The MCF approach allows for repair of the entire tegmen since most patients have multiple skull base defects. This multi-layer repair of the entire tegmen may allow for a long-term durable repair. In addition, the MCF repair also avoids disruption of the ossicular chain. The MCF approach is highly effective with a very low recurrence rate both in this study and in review of previous published studies(1). Finally, we demonstrate the MCF approach results in significant improvement in audiologic outcomes in a cohort of 27 ears where $56 \%$ of patients had multiple tegmen defects and $82 \%$ of patients had encephaloceles. We found that $96.3 \%$ of ears showed improvement in $A B G$ with only 1 patient demonstrating no change in $A B G$ and only 1 patient showing slightly worse WDS (76\% postoperatively vs. $80 \%$ preoperatively). No patient had recurrence of CSF leak.

The MCF approach is the preferred approach to repair SCSF leaks in prior studies(1), but a comparison of the audiologic outcomes reported here to those reported for the transmastoid (TM) approach(11) or the combined TM-MCF approach(12,13) is warranted. Kim et al described results of 16 patients using the TM approach, where the ossicular chain was not disrupted(11). Only $31 \%$ of patients in their study had multiple tegmen defects, while enephaloceles were found in $81 \%$ of patients. There was improvement in PTA in 15 of 16 patients and worsening of WDS scores in 3 of 16 patients. One patient had recurrence and required MCF approach. Son et al described the TM-MCF approach in 19 patients where 3 patients experience $>15 \mathrm{~dB}$ sensorineural hearing loss likely from manipulation of the ossicular chain(13). Stevens et al described the TM-MCF approach in 28 patients (31 ears), where the incus-stapes joint is disarticulated prior to skull base repair(12). Encephaloceles were identified in $77 \%$ of cases. While the ABG improved, the PTA improvement was not statistically 
significant. Worsening of the PTA or ABG was noted in $35 \%$ of cases. It is possible that disruption of the ossicular chain contributes to less optimal hearing outcomes with the TM-MCF approach.

The underlying mechanism of the conductive hearing loss in patients with spontaneous CSF leaks is from the presence of middle ear effusion and potentially from herniated encephaloceles contacting the ossicular chain, thereby dampening sound transmission $(11,14,15)$. As mentions above, the rate of encephalocele in patients with sCSF leaks ranges from $77-82 \%(11,12)$. Removal of the encephalocele and sealing the CSF leak to resolve the effusion results in consistent improvement in audiologic outcomes.

The mechanistic etiology of temporal sCSF leaks is still not definitively understood. There is a clear association with obesity, middle age and female gender(1). sCSF leak patients not only have obligate tegmen bone defects but also have $22 \%$ thinner calvaria compared to obese controls(3). This bone thinning is isolated to the skull, because the extracranial zygoma bone is not affected(3). This suggests that an intracranial process leads to skull and skull base thinning. Elevated intracranial pressure, either chronically (idiopathic intracranial hypertension, $\mathrm{IIH}$ ) or transiently (during obstructive sleep apnea(16)) could lead to skull and skull base thinning(1). The incidence of IIH in SCSF leak patients is between $35 \%(17)$ and $67 \%(18)$ and the incidence of OSA in SCSF leaks patients is $83 \%(4)$. OSA is independently associated with thinning of the skull and skull base(5).

We perform surgical repair of SCSF leaks to reduce or eliminate the risk of meningitis. The rate of preoperative meningitis is about $20 \%$ and ranges from $6 \%(13)$ to $58 \%(19)$. Additional reasons for repair include improvement in hearing and resolving chronic otorrhea. Given the high success rate and low morbidity of sCSF leak repair, we believe the benefits and risks of repair outweigh the complications of meningitis. After repair, we advise all patients to obtain sleep study in the perioperative period. Patients are also advised to obtain LP for ICP measurement, but not all patients comply. Patients who have an elevated ICP on LP are trialed 
on acetazolamide and/or offered VP shunt. We typically do not obtain ophthalmology consult for papilledema unless the patient complains of vision changes based on a study that showed a very low rate of papilledema in SCSF leak patients(20).

The limitations of our study include the small sample size, retrospective study design, short follow-up duration, and variable timing of postoperative audiometric assessments. To address these limitations, future endeavors should be based on prospective, multi-center studies with fixed time points at which postoperative audiometric assessments should be performed. Despite these limitations, this is the first report of audiologic outcomes following MCF only approach for repair of temporal sCSF leaks.

The results of our study demonstrate that the MCF approach for repair of spontaneous CSF leaks yields significant improvement in conductive hearing loss. Therefore, not only is the MCF approach a reliable, safe, and effective way to manage clear otorrhea in patients with spontaneous CSF leaks, the resulting hearing loss from spontaneous CSF leaks can also be effectively and safely managed using this approach. 
CSF Leaks and Hearing Outcomes

\section{REFERENCES}

1. Lobo BC, Baumanis MM, Nelson RF. Surgical repair of spontaneous cerebrospinal fluid (CSF) leaks: A systematic review. Laryngoscope investigative otolaryngology 2017;2:215-24.

2. Nelson RF, Gantz BJ, Hansen MR. The rising incidence of spontaneous cerebrospinal fluid leaks in the United States and the association with obesity and obstructive sleep apnea. Otol Neurotol 2015;36:476-80.

3. Nelson RF, Hansen KR, Gantz BJet al. Calvarium thinning in patients with spontaneous cerebrospinal fluid leak. Otol Neurotol 2015;36:481-5.

4. Rabbani CC, Saltagi MZ, Manchanda SKet al. Prevalence of Obstructive Sleep Apnea (OSA) in Spontaneous Cerebrospinal Fluid (CSF) Leaks: A Prospective Cohort Study. Otol Neurotol 2018;39:e475-e80.

5. Rabbani C, Saltagi MZ, Ye MJet al. Association of Obstructive Sleep Apnea With Calvarial and Skull Base Thinning. JAMA otolaryngology-- head \& neck surgery 2018;144:513-8.

6. El Hadi T, Sorrentino T, Calmels MNet al. Spontaneous tegmen defect and semicircular canal dehiscence: same etiopathogenic entity? Otol Neurotol 2012;33:591-5.

7. Goddard JC, Meyer T, Nguyen Set al. New considerations in the cause of spontaneous cerebrospinal fluid otorrhea. Otol Neurotol 2010;31:940-5.

8. Nelson RF, Roche JP, Gantz BJet al. Middle Cranial Fossa (MCF) Approach Without the Use of Lumbar Drain for the Management of Spontaneous Cerebral Spinal Fluid (CSF) Leaks. Otol Neurotol 2016;37:1625-9.

9. Gurgel RK, Jackler RK, Dobie RAet al. A new standardized format for reporting hearing outcome in clinical trials. Otolaryngol Head Neck Surg 2012;147:803-7.

10. Wick CC, Killeen DE, Clark Met al. Posterior Fossa Spontaneous Cerebrospinal Fluid Leaks. Otol Neurotol 2017;38:66-72.

11. Kim L, Wisely CE, Dodson EE. Transmastoid approach to spontaneous temporal bone cerebrospinal fluid leaks: hearing improvement and success of repair. Otolaryngol Head Neck Surg 2014;150:472-8.

12. Stevens SM, Crane R, Pensak MLet al. Analysis of Audiometric Outcomes following Combined Middle Cranial Fossa/Transmastoid Approaches for Spontaneous Cerebrospinal Fluid Otorrhea. Otolaryngol Head Neck Surg 2017;156:924-32.

13. Son HJ, Karkas A, Buchanan Pet al. Spontaneous cerebrospinal fluid effusion of the temporal bone: repair, audiological outcomes, and obesity. Laryngoscope 2014;124:1204-8.

14. Lundy LB, Graham MD, Kartush JMet al. Temporal bone encephalocele and cerebrospinal fluid leaks. Am J Otol 1996;17:461-9.

15. Semaan MT, Gilpin DA, Hsu DPet al. Transmastoid extradural-intracranial approach for repair of transtemporal meningoencephalocele: a review of 31 consecutive cases. Laryngoscope 2011;121:1765-72.

16. Jennum P, Borgesen SE. Intracranial pressure and obstructive sleep apnea. Chest 1989;95:27983.

17. Allen KP, Perez CL, Kutz JWet al. Elevated intracranial pressure in patients with spontaneous cerebrospinal fluid otorrhea. Laryngoscope 2014;124:251-4.

18. Brainard L, Chen DA, Aziz KMet al. Association of benign intracranial hypertension and spontaneous encephalocele with cerebrospinal fluid leak. Otol Neurotol 2012;33:1621-4.

19. Markou K, Goudakos J, Franco-Vidal Vet al. Spontaneous osteodural defects of the temporal bone: diagnosis and management of 12 cases. American journal of otolaryngology 2011;32:13540. 
CSF Leaks and Hearing Outcomes

20. Aaron G, Doyle J, Vaphiades MSet al. Increased intracranial pressure in spontaneous CSF leak patients is not associated with papilledema. Otolaryngol Head Neck Surg 2014;151:1061-6. 


\section{FIGURE LEGENDS}

Figure 1: Representative coronal CT image of preoperative tegmen dehiscence (arrow) and middle ear fluid signal. Postoperative coronal CT image of same patient after repair with Hydroset bone substitute (arrow). Not absence of middle ear fluid in postoperative image.

Figure 2: Pre-treatment audiometric scattergram demonstrating pure tone average and word recognition score in the study cohort

Figure 3: Post-treatment audiometric scattergram demonstrating changes in pure tone average and word recognition scores following middle cranial fossa approach 